\title{
Efficacy and toxicity of treatment with the anti-CTLA-4 antibody ipilimumab in patients with metastatic melanoma after prior anti-PD-1 therapy
}

\begin{abstract}
S Bowyer ${ }^{1,2}$, P Prithviraj ${ }^{3,4}$, P Lorigan $^{5}$, J Larkin ${ }^{6}, \mathrm{G} \mathrm{McArthur}^{7}, \mathrm{~V}_{\text {Atkinson }}{ }^{8}, \mathrm{M} \mathrm{Millward}^{2,9}, \mathrm{M} \mathrm{Khou}^{10}, \mathrm{~S} \mathrm{Diem}^{6}$, S Ramanujam ${ }^{11}$, B Kong ${ }^{10}$, E Liniker ${ }^{11}$, A Guminski ${ }^{11}$, P Parente ${ }^{12}$, M C Andrews ${ }^{3,4}$, S Parakh ${ }^{3}, J_{\text {Cebon }}^{3,4}$, G V Long ${ }^{11,13}$, M S Carlino ${ }^{10,11,13}$ and O Klein ${ }^{\star, 3,4}$

${ }^{1}$ Rockingham General Hospital, Cooloongup, Western Australia, Australia; ${ }^{2}$ School of Medicine and Pharmacology, University of Western Australia, Nedlands, Western Australia, Australia; ${ }^{3}$ Olivia Newton- John Cancer Centre, Austin Hospital, Heidelberg, Melbourne, Victoria, Australia; ${ }^{4}$ Olivia Newton-John Cancer Research Institute, Heidelberg, Melbourne, Victoria, Australia; ${ }^{5}$ The Christie NHS Foundation Trust and University of Manchester, Manchester, UK; ${ }^{6}$ Royal Marsden Hospital NHS Foundation Trust, London, UK; ${ }^{7}$ Peter MacCallum Cancer Centre, Melbourne, Victoria, Australia; ${ }^{8}$ Princess Alexandra Hospital, Greenslopes Private Hospital, Brisbane, Queensland, Australia; ${ }^{9}$ Department of Medical Oncology, Sir Charles Gairdner Hospital, Nedlands, Western Australia, Australia; ${ }^{10}$ Westmead Hospital, Sydney, New South Wales, Australia; ${ }^{11}$ Melanoma Institute Australia, Sydney, New South Wales, Australia; ${ }^{12}$ Box Hill Hospital, Box Hill, Victoria, Australia and ${ }^{13}$ University of Sydney, Sydney, New South Wales, Australia
\end{abstract}

Background: Recent phase III clinical trials have established the superiority of the anti-PD-1 antibodies pembrolizumab and nivolumab over the anti-CTLA-4 antibody ipilimumab in the first-line treatment of patients with advanced melanoma. Ipilimumab will be considered for second-line treatment after the failure of anti-PD-1 therapy.

Methods: We retrospectively identified a cohort of 40 patients with metastatic melanoma who received single-agent anti-PD-1 therapy with pembrolizumab or nivolumab and were treated on progression with ipilimumab at a dose of $3 \mathrm{mg} \mathrm{kg}^{-1}$ for a maximum of four doses.

Results: Ten percent of patients achieved an objective response to ipilimumab, and an additional $8 \%$ experienced prolonged ( $>6$ months) stable disease. Thirty-five percent of patients developed grade 3-5 immune-related toxicity associated with ipilimumab therapy. The most common high-grade immune-related toxicity was diarrhoea. Three patients (7\%) developed grade 3-5 pneumonitis leading to death in one patient.

Conclusions: Ipilimumab therapy can induce responses in patients who fail the anti-PD-1 therapy with response rates comparable to previous reports. There appears to be an increased frequency of high-grade immune-related adverse events including pneumonitis that warrants close surveillance.

Understanding the molecular basis of immune checkpoint inhibition is transforming the management of metastatic melanoma, and is likely to have a major impact on other cancers
(Hamid et al, 2013). Ipilimumab, a monoclonal antibody that blocks cytotoxic T-lymphocyte-associated antigen 4 (CTLA-4) to potentiate anti-tumour $\mathrm{T}$-cell responses, has been shown to

*Correspondence: Dr O Klein; E-mail: oliver.klein@onjcri.org.au

Received 26 November 2015; revised 2 March 2016; accepted 14 March 2016; published online 28 April 2016

(c) 2016 Cancer Research UK. All rights reserved 0007-0920/16 
prolong overall survival (Hodi et al, 2010; Robert et al, 2011), with a proportion of patients surviving beyond 5 years (Schadendorf et al, 2015). The main toxicity seen with ipilimumab is related to activation of the immune system against autoantigens. Grade 3-4 immune-related adverse events (irAEs) occur in 10\%-15\% of patients: primarily skin rash, diarrhoea and colitis, hepatitis and endocrinopathies (Hodi et al, 2010; Robert et al, 2011). Ipilimumab is approved by regulatory authorities for the first- and subsequentline treatment of metastatic melanoma.

The programmed cell death 1 (PD-1) receptor negatively regulates the effector phase of $\mathrm{T}$-cell responses after binding to PD-L1, which is frequently expressed on tumour cells and in the tumour microenvironment (Rotte et al, 2015). Antibodies blocking this interaction release the cytotoxic potential of tumour-specific T-cells. Pembrolizumab and nivolumab, monoclonal antibodies that bind the PD-1 receptor, have demonstrated significant and durable tumour responses with manageable toxicity profiles (Hamid et al, 2013; Topalian et al, 2014; Robert et al, 2015a).

Two recent phase III studies have reported the superiority of both pembrolizumab and nivolumab to ipilimumab in patients with advanced melanoma as first-line therapy (Larkin et al, 2015; Robert et al, 2015b). The Keynote 006 study (NCT01866319) randomised 834 patients in a $1: 1: 1$ ratio to receive ipilimumab or two dosing schedules of pembrolizumab. The estimated 6-month progression-free survival (PFS) rate was significantly higher for both pembrolizumab arms as compared with ipilimumab $(47.3 \%$, $46.4 \%$, 26.5\%, respectively) with estimated 12-month overall survival rates of $74.1 \%, 68.4 \%, 58.2 \%$, respectively (Robert et al, 2015b). The Checkmate 067 study (NCT01844505) evaluated nivolumab or nivolumab plus ipilimumab vs ipilimumab alone in 945 patients with previously untreated advanced melanoma. Progression-free survival was significantly longer for nivolumab alone or combined with ipilimumab compared with ipilimumab alone (6.9 months, 11.5 months, 2.9 months, respectively) and objective response rates higher $(43.7 \%, 57.6 \%, 19.0 \%$, respectively; Larkin et al, 2015). On the basis of these results, anti-PD-1 antibodies represent a new standard of care in the first-line treatment of patients with advanced melanoma.

Combination immunotherapy with nivolumab and ipilimumab is associated with higher objective response rates and PFS compared with monotherapy ipilimumab. Combination therapy, however, is associated with significant toxicity with greater than half of patients experiencing grade 3 or 4 adverse events (Larkin et al, 2015). It is currently unclear whether combined anti-PD-1 antibody with ipilimumab treatment will be widely adopted due to concerns about toxicity but the doublet is currently licensed by the US Food and Drug Administration. In addition, the overall survival data are not yet reported and this study was not designed to compare the combination vs nivolumab monotherapy for this end point (Larkin et al, 2015). For patients who have progressed on anti-PD-1 therapy, ipilimumab will be a frequent second- or thirdline treatment. Currently, there is very limited evidence regarding the efficacy and toxicity of ipilimumab after anti-PD-1 antibodies. We report the clinical efficacy and toxicity profile seen with this therapeutic sequence in the largest retrospective cohort to date.

\section{MATERIALS AND METHODS}

Patient population. We retrospectively identified patients with advanced melanoma who had sequentially been treated with the anti-PD-1 antibodies pembrolizumab or nivolumab followed by ipilimumab. Only patients who had received sequential treatment were suitable for inclusion. Cases were identified at eight sites in Australia and two in the United Kingdom. The data collected included: baseline demographics (age, gender), mutational status
(BRAF/NRAS) and prognostic variables (American Joint Committee on Cancer (AJCC) stage of disease, lactate dehydrogenase (LDH), Eastern Cooperative Oncology Group (ECOG) performance status); details of the anti-PD-1 inhibitor treatment (type, dosage, number of cycles received and toxicity) and ipilimumab treatment (number of cycles and toxicity); data pertaining to response to respective therapies; time interval between PD-1 inhibitor treatment and commencement of ipilimumab; and time to end point data. Progression-free survival was defined as the time between date of commencement of therapy to date of progression or death. The response assessments were assessed according to response evaluation criteria in solid tumors (RECIST) 1.1 (Eisenhauer et al, 2009) and/or immune-related response criteria (Wolchok et al, 2009). Severity of adverse events was graded according to the National Cancer Institute Common Terminology Criteria for Adverse Events (CTCAE) v4.0.

The identified patients all accessed anti-PD-1 therapy through clinical trials and the schedule and dose received was as per clinical trial protocol. All the sites had the appropriate ethics approval for the relevant clinical trial. Subsequent ipilimumab treatment was at standard dosing ( $3 \mathrm{mg} \mathrm{kg}^{-1}$ intravenously, three weekly to a maximum of four doses).

\section{RESULTS}

Patient demographics and anti-PD-1 therapy. A total of 40 patients were identified who received either pembrolizumab or nivolumab followed by ipilimumab between November 2012 and July 2015. The baseline demographic data are detailed in Table 1, and are consistent with typical melanoma patient populations, except for the low rate of BRAF mutation positivity (10\%), likely due to selection/eligibility for anti-PD-1 studies. The majority of

Table 1. Baseline characteristics before anti-PD-1 therapy

\begin{tabular}{|c|c|}
\hline & Total (\%); $N=40$ \\
\hline Age in years, median (range) & $61(37-79)$ \\
\hline \multicolumn{2}{|l|}{ Sex } \\
\hline Female & $15(37)$ \\
\hline Male & $25(63)$ \\
\hline \multicolumn{2}{|l|}{ Mutational status } \\
\hline BRAF V600 mutated & $4(10)$ \\
\hline Wild-type & $36(90)$ \\
\hline \multicolumn{2}{|l|}{ AJCC stage } \\
\hline M1a & $2(5)$ \\
\hline M1b & $4(10)$ \\
\hline M1c & $34(85)$ \\
\hline \multicolumn{2}{|c|}{ Pre-treated brain metastases at treatment } \\
\hline No & $37(92)$ \\
\hline Yes & $3(8)$ \\
\hline \multicolumn{2}{|l|}{ ECOG performance status } \\
\hline $0-1$ & $40(100)$ \\
\hline$\geqslant 2$ & 0 \\
\hline \multicolumn{2}{|l|}{ LDH } \\
\hline Normal & $24(60)$ \\
\hline Elevated & $16(40)$ \\
\hline \multicolumn{2}{|l|}{ Prior therapy } \\
\hline BRAF $+/-$ MEK inhibitor & $3(7)$ \\
\hline Ipilimumab & $4(10)$ \\
\hline Chemotherapy & 7 (17) \\
\hline Other & $2(5)^{a}$ \\
\hline Nil & $26(64)$ \\
\hline
\end{tabular}


patients were males (63\%) with AJCC stage M1c disease (85\%). All patients had a good performance status before commencement of anti-PD-1 therapy (ECOG 0-1) and 60\% had a normal baseline $\mathrm{LDH}$. Thirty-six percent of patients had received other systemic therapy before the commencement of a PD-1 inhibitor. Four (10\%) patients had received ipilimumab as a prior therapy and were subsequently rechallenged.

Of the 40 patients identified, 26 (65\%) received pembrolizumab at either a dose of $10 \mathrm{mg} \mathrm{kg}^{-1}$ on a two or three weekly basis or $2 \mathrm{mg} \mathrm{kg}^{-1}$ three weekly and 14 (35\%) patients received nivolumab $3 \mathrm{mg} \mathrm{kg}^{-1}$ two weekly. All patients discontinued anti-PD 1 inhibitor owing to progression and were treated with ipilimumab as the next line of systemic therapy.

The median treatment duration on anti-PD-1 therapy and median PFS were 5 months. Eight (20\%) patients achieved an objective response to anti-PD-1 therapy, 15 (38\%) had stable disease and $17(42 \%)$ had progressive disease as their best treatment response (Table 2$)$. Three $(8 \%)$ patients developed grade $3 / 4$ irAEs thought attributable to the PD- 1 inhibitor (Table 3 ).

Efficacy and toxicity of ipilimumab following failure of antiPD-1 therapy. The median follow-up for all patients after commencement of ipilimumab therapy was 12.5 months (range 4-23 months). Four (10\%) patients achieved an objective response to ipilimumab, and an additional 3 (8\%) experienced prolonged stable disease (greater than 6 months) after completion of their treatment. Thirty-three (82\%) patients progressed on therapy (Table 2). Of the patients who obtained a clinical benefit (objective response or prolonged stable disease $>6$ months) from ipilimumab, one (1/17) had previously failed to respond to anti-PD-1 therapy, three patients had stable disease and an additional three had a partial response as their best treatment response to anti-PD1 therapy. Two patients experiencing a clinical benefit experienced grade $3 / 4$ irAEs. The median interval between the final dose of PD-1 inhibitor therapy and the first dose of ipilimumab was 53 days (range 2-683 days). The median time between the last dose of anti-PD-1 therapy and the commencement of ipilimumab therapy did not significantly differ between patients obtaining a clinical benefit from ipilimumab and non-responders. The median interval between therapies was 32 days for those who experienced toxicity. Only $22(55 \%)$ patients received all the four doses of ipilimumab,

Table 2. Response to treatment

\begin{tabular}{|l|c|c|}
\hline Best overall response-no. (\%) & $\begin{array}{c}\text { Pembrolizumab, } \\
\mathbf{N}=\mathbf{2 6}(\mathbf{6 5 \%}) \\
\text { Nivolumab, } \\
\mathbf{N = 1 4}(\mathbf{3 5 \%})\end{array}$ & Ipilimumab, \\
$\mathbf{N}=\mathbf{4 0}$ \\
\hline Complete response & 0 & 0 \\
Partial response & $8(20)$ & $4(10)$ \\
Stable disease & $15(38)$ & $3(8)$ \\
Progressive disease & $17(42)$ & $33(82)$ \\
\hline
\end{tabular}

Table 3. Severe immune-related adverse events

\begin{tabular}{|c|c|c|}
\hline & $\begin{array}{c}\text { Pembrolizumab, } \\
N=26(65 \%) \\
\text { Nivolumab, } \\
N=14(35 \%)\end{array}$ & $\begin{array}{l}\text { Ipilimumab, } \\
\qquad=\mathbf{4 0}\end{array}$ \\
\hline Grade 3/4 irAEs-no. (\%) & $\begin{array}{c}3(8) \\
\text { Hypothyroidism-1 } \\
\text { Panhypopituitarism-1 } \\
\text { Bullous pemphigoid-1 }\end{array}$ & $\begin{array}{c}14(35 \%) \\
\text { Colitis/diarrhoea-9 } \\
\text { Pneumonitis-3 } \\
\text { Hepatitis-2 } \\
\text { Encephalitis-1 }\end{array}$ \\
\hline
\end{tabular}

with early termination owing to toxicity (30\%) and/or clinical deterioration (15\%).

Fourteen (35\%) patients developed grade 3-5 irAEs associated with ipilimumab therapy (Table 3 ). Ten percent of these highgrade irAEs occurred after receiving only one cycle of ipilimumab. No patients who were rechallenged with ipilimumab experienced a high-grade irAE, and only one of the five patients achieved an objective response. The most common high-grade irAE was diarrhoea or colitis (grade $3 / 423 \%$ ), all requiring immunosuppressive therapies. Other grade $3 / 4$ immune-related toxicities were hepatitis, pneumonitis and a case of encephalitis. Two (5\%) patients experienced two grade 3/4 iAEs simultaneously (pneumonitis and hepatitis, diarrhoea and hepatitis), which required multiple lines of immunosuppression before improvement was seen clinically and biochemically. Three (8\%) patients developed grade $3-5$ pneumonitis, one of whom died (Figure 1). One patient recovered fully after a prolonged course of high-dose intravenous and oral steroids and the third patient required $>6$ months of immunosuppression with oral steroids, mycophenolate and tacrolimus. Two of the 14 (14\%) patients who experienced high-grade irAEs with ipilimumab had developed severe irAEs (hypothyroidism, bullous pemphigoid) with previous anti-PD-1 therapy.

\section{DISCUSSION}

Recent clinical trials have demonstrated superior efficacy of the anti-PD-1 antibodies pembrolizumab and nivolumab compared with ipilimumab in the front line treatment of patients with advanced melanoma establishing these agents as a new standard of care (Larkin et al, 2015; Robert et al, 2015b).Thirty to forty-five percent of patients respond to anti-PD-1 therapy with durable responses in the majority. Nevertheless, a significant proportion of patients will require second-line therapy, and ipilimumab is the most likely choice. Although the safety profile for pembrolizumab in ipilimumab naive and pre-treated patients was similar in the KEYNOTE-001 study (Daud et al, 2015), and nivolumab has been tolerated safely as sequential treatment, even after patients have experienced grade 3 toxicity with ipilimumab (Weber et al, 2013; Wolchok et al, 2013), there is very limited evidence for the reverse treatment sequence.

We report on a large cohort of patients that have received treatment with ipilimumab after failure of anti-PD-1 therapy. Twenty percent of patients initially obtained an objective response to anti-PD-1 therapy, a lower response rate than reported in monotherapy clinical trials. Responses to anti-PD-1 therapy are usually durable; as this patient cohort was retrospectively identified as requiring subsequent ipilimumab, it is expected the response rates would be lower than that in an unselected population. The biology of innate $v s$ acquired resistance to anti-PD-1 therapy is likely to be different. Innate resistance to anti-PD-1 therapy, as seen in the majority of this cohort, may be a poor prognostic variable or may indicate that alternate approaches to immune modulation need to be considered.

Twenty percent of patients in our cohort obtained a clinical benefit (response or prolonged stable disease) from ipilimumab therapy in keeping with previous reports in patients who have progressed on anti-PD-1 therapy (Weber et al, 2013). The objective response rate of $10 \%$ is similar to the response rates seen with ipilimumab in trials in the first- and second-line setting (Hodi et al, 2010; Larkin et al, 2015; Robert et al, 2015b). However, of patients who progressed through anti-PD-1 inhibitor therapy, only one responded to subsequent ipilimumab. Overall survival data for this cohort is immature. Ipilimumab can induce durable responses in patients who achieve stable disease, as well as those who achieve an 

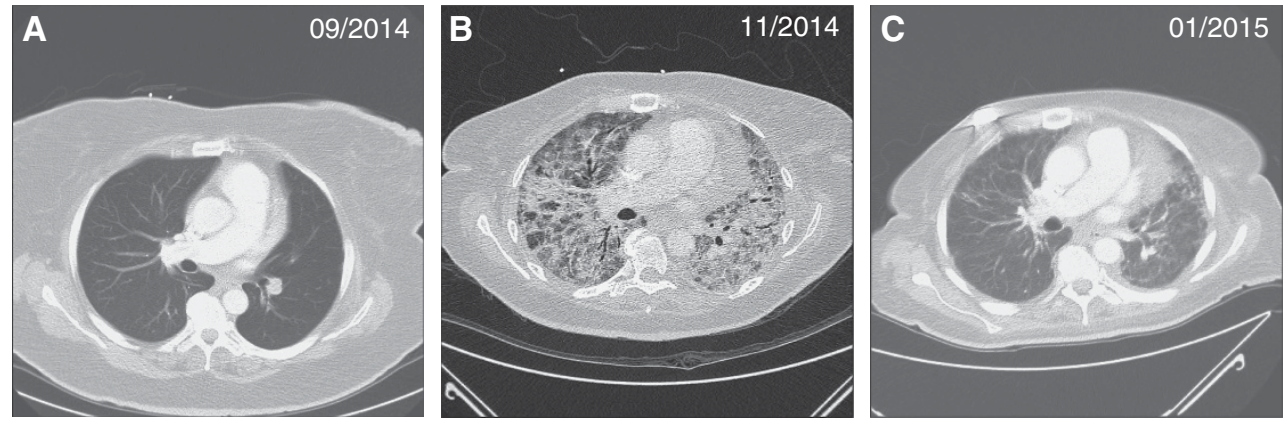

Figure 1. Computer tomography scans demonstrating a severe pneumonitis during ipilimumab therapy; a 61-year-old patient with metastatic melanoma commenced ipilimumab after having progressed several weeks earlier on nivolumab treatment (A). She developed a severe pneumonitis after three doses of ipilimumab (B) that resolved on intensified immunosuppression with a prolonged course of high dose intravenous and oral glucocorticoids (C).

objective response making overall survival a more meaningful end point to assess the efficacy of ipilimumab (Schadendorf et al, 2015). The authors acknowledge that response rates alone are insufficient to make conclusions on the efficacy of ipilimumab after failure of anti-PD-1 therapy and further prospective studies are required. However, responses to ipilimumab can be seen even after failure of anti-PD-1 therapy making it a viable treatment option.

An important finding in this analysis is that grade $3 / 4$ immunerelated toxicity occurred in over a third of patients treated with sequential ipilimumab following anti-PD-1 therapy, a higher rate than generally observed with the currently approved dose of $3 \mathrm{mg} \mathrm{kg}^{-1}$ ipilimumab (Hodi et al, 2010; Kaufman et al, 2013; Robert et al, 2015b). Also, increased toxicity did not appear to correlate with improved response rates, unlike previous reports in the literature (Downey et al, 2007). Diarrhoea or colitis were the most common toxicities, followed by hepatitis, consistent with the known toxicity profile of ipilimumab. Gastrointestinal and hepatic toxicity typically develops 6-7 weeks following the commencement of ipilimumab (Weber et al, 2012); however, we encountered early toxicity, as three patients experienced grade 3 diarrhoea after one cycle of ipilimumab. In this cohort, three patients experienced grade $3 / 4$ pneumonitis, and one patient died due to this treatment complication. Pneumonitis has been rarely described with ipilimumab therapy and is more commonly reported in association with PD-1 inhibitors (Weber et al, 2015). Our experience suggests that it is a more frequent irAE encountered with ipilimumab delivered subsequent to anti-PD-1 therapy.

Pharmacokinetic studies have demonstrated that the mean elimination half-life of pembrolizumab is 26 days and 17-25 days for nivolumab, with linear pharmacokinetics (Bristol-Myer Squibb, 2013; Merck, 2014). In addition, flow cytometry has been used to estimate PD-1 occupancy on circulating T cells over time with nivolumab. Owing to its high affinity, a mean plateau occupancy of $72 \%$ was seen for $\geqslant 59$ days after a single dose, suggesting that even when serum levels are undetectable sufficient concentrations persist to maintain PD-1 occupancy (Brahmer et al, 2010). The high rates of toxicity in this group with a median time between treatments of 32 days may indicate that this is not an adequate washout period.

In addition, biomarker studies from a phase I study of nivolumab demonstrated that CTLA-4 positive CD4 T cells were significantly increased in non-responders after treatment which could be a potentiating factor for the excess toxicity seen in this series given that all patients discontinued anti-PD-1 therapy due to progression (Gibney et al, 2015).

Translational studies using blood samples collected from patients who have been treated with anti-PD-1 or anti-CTLA-4 agents alone, in combination or sequenced have revealed vastly different T-cell gene expression profiles between these patient cohorts (Das et al, 2015). This underscores that a combined blockade of co-inhibitory T-cell receptors at the same time can lead to a different immunological outcome than the sequential engagement of the same receptors. A more detailed understanding of such dose, sequence and combinatorial differences on immune and tumour cell function is urgently required to guide selection of the most mechanistically rational immunotherapy regimen. Furthermore, the immunological profile induced by anti-PD-1 pre-treatment may increase the autoimmune potential of $\mathrm{T}$ cells that are subsequently activated during anti-CTLA-4 therapy and hence be a mechanism for increased toxicity.

In keeping with our observation of increased toxicity of anti-CTLA-4 blockade after anti-PD-1 treatment, a case series reported on three patients who were treated in the same sequence and experienced prolonged, difficult-to-treat autoimmune colitis (Danlos et al, 2015). A further series of patients treated with sequential therapy reported that three out of five patients experienced high-grade irAEs, one of which was encephalopathy in addition to hepatitis (Khoja et al, 2015). There is also some suggestion that toxicity of vemurafenib may be potentiated by prior anti-PD-1 therapy (Johnson et al, 2013; Khoja et al, 2015).

Results of pivotal phase III studies have established PD-1 inhibitors as first-line therapy in advanced melanoma. For those failing anti-PD-1 therapy, ipilimumab will be a treatment option for many patients, as borne out by current clinical practice in patients progressing on PD-1 inhibitors and owing to a lack of effective therapeutic alternatives outside of clinical trials. However, our early experience suggests that the toxicity of ipilimumab given after anti-PD-1 therapy may be greater than is seen in first-line therapy or after other treatments. For the moment, when treating with ipilimumab following failure of PD-1 inhibitors, oncologists should have increased vigilance for severe and potentially early immune-related toxicity.

Many questions remain unanswered surrounding appropriate patient selection and predictive biomarkers for immune checkpoint inhibitors in clinical practice. Greater understanding of the tumour microenvironment and the process of immune-editing is fundamental to allow rational stratification of new immunotherapies and combinations to achieve optimal responses on an individual basis (Teng et al, 2015).

\section{CONFLICT OF INTEREST}

P Lorigan received consultancy and honoraria from Bristol Myer Squibb, Glaxosmithkline, Amgen, Novartis, Roche, Merck. J Larkin received institutional research support from MSD, Bristol Myer 
Squibb, Pfizer and Novartis; non-remunerated consultancy for Glaxosmithkline, Novartis, MSD, Bristol Myer Squibb, Pfizer and Roche/Genentech. G McArthur received research grant support from Pfizer, Celgene, Ventana; consultancy for Provectus; uncompensated consultancy for Pfizer, Millenium, Glaxosmithkline, Roche/Genentech, Novartis, Bristol Myer Squibb and Amgen. V Atkinson participated in an advisory committee for MSD; was a member of the advisory board for Bristol Myer Squibb; received honoraria from GSK and received travel support from GSK, BMS and Roche. M Millward received consultancy or advisory role to Roche, Bristol Myer Squibb, MSD and Glaxosmithkline; received research funding from Roche and Glaxosmithkline. S Ramanujam received travel and accommodation grants from Amgen. MC Andrews received travel support from Bristol Myer Squibb and MSD. J Cebon participated in advisory boards for Amgen and Merck. GV Long participated in advisory boards of Amgen, Bristol Myer Squibb, Glaxosmithkline, Novartis, Provectus, Roche and Merck Inc. MS Carlino participated in advisory boards for Merck, Amgen, Novartis and Bristol Myer Squibb. The remaining authors declare no conflict of interest.

\section{REFERENCES}

Brahmer JR, Drake CG, Wollner I, Powderly JD, Picus J, Sharfman WH, Stankevich E, Pons A, Salay TM, McMiller TL, Gilson MM, Wang C, Selby M, Taube JM, Anders R, Chen L, Korman AJ, Pardoll DM, Lowy I, Topalian SL (2010) Phase I study of single-agent anti-programmed death-1 (MDX-1106) in refractory solid tumors: safety, clinical activity, pharmacodynamics, and immunologic correlates. J Clin Oncol 28(19): 3167-3175

Bristol-Myer Squibb (2013) Investigator Brochure NIVOLUMAB BMS936558 MDX1106 Version 12.

Danlos FX, Pagès C, Roux J, Jebali M, Gornet JM, Bagot M, Lebbé C (2015) Atypical severe immune-related adverse effects resulting from sequenced immunotherapy in melanoma. Melanoma Res 25: 178-179.

Das R, Verma R, Sznol M, Boddupalli CS, Gettinger SN, Kluger H, Callahan M, Wolchok JD, Halaban R, Dhodapkar MV, Dhodapkar KM (2015) Combination therapy with anti-CTLA-4 and anti-PD-1 leads to distinct immunologic changes in vivo. J Immunol 194: 950-959.

Daud A, Ribas A, Robert C, Hodi S, Wolchok JD, Joshua AM, Hwu WJ, Weber JS, Gangadhar TC, Joseph RW, Dronca RS, Patnaik A, Zarour HM, Kefford R, Lindia JA, Li XN, Ebbinghaus S, Kang SP, Hamid O (2015) Long-term efficacy of pembrolizumab (pembro; MK-3475) in a pooled analysis of 655 patients (pts) with advanced melanoma (MEL) enrolled in KEYNOTE-001. J Clin Oncol 33(Suppl): abstr 9005.

Downey SG, Klapper JA, Smith FO, Yang JC, Sherry RM, Royal RE, Kammula US, Hughes MS, Allen TE, Levy CL, Michael Y, Nichol G, White DE, Steinberg SM, Rosenberg SA (2007) Prognostic factors related to clinical response in patients with metastatic melanoma treated by CTL-associated antigen-4 blockade. Clin Cancer Res 13(1): 6681-6688.

Eisenhauer EA, Therasse P, Bogaerts J, Schwartz LH, Sargent D, Ford R, Dancey J, Arbuck S, Gwyther S, Mooney M, Rubinstein L, Shankar L, Dodd L, Kaplan R, Lacombe D, Verweij J (2009) New response evaluation criteria in solid tumours: revised RECIST guideline (version 1.1). Eur J Cancer 45(2): 228-247.

Gibney GT, Kudchadkar RR, DeConti RC, Thebeau MS, Czupryn MP, Tetteh L, Eysmans C, Richards A, Schell MJ, Fisher KJ, Horak CE, Inzunza HD, Yu B, Martinez AJ, Younos I, Weber JS (2015) Safety, correlative markers, and clinical results of adjuvant nivolumab in combination with vaccine in resected high-risk metastatic melanoma. Clin Cancer Res 21(4): $712-720$.

Hamid O, Robert C, Daud A, Hodi FS, Hwu WJ, Kefford R, Wolchok JD, Hersey P, Joseph RW, Weber JS, Dronca R, Gangadhar TC, Patnaik A, Zarour H, Joshua AM, Gergich K, Elassaiss-Schaap J, Algazi A, Mateus C, Boasberg P, Tumeh PC, Chmielowski B, Ebbinghaus SW, Li XN, Kang SP, Ribas A (2013) Safety and tumor responses with lambrolizumab (anti-PD-1) in melanoma. $N$ Engl J Med 369(2): 134-144.

Hodi FS, O'Day SJ, McDermott DF, Weber RW, Sosman JA, Haanen JB, Gonzalez R, Robert C, Schadendorf D, Hassel JC, Akerley W, van den Eertwegh AJM, Lutzky J, Lorigan P, Vaubel JM, Linette GP, Hogg D,
Ottensmeier CH, Lebbé C, Peschel C, Quirt I, Clark JI, Wolchok JD, Weber JS, Tian J, Yellin MJ, Nichol GM, Hoos A, Urba WJ (2010) Improved survival with ipilimumab in patients with metastatic melanoma. N Engl J Med 363(8): 711-723.

Johnson DB, Wallender EK, Cohen DN, Likhari SS, Zwerner P, Powers JG, Shinn L, Kelley MC, Joseph RW, Sosman JA (2013) Severe cutaneous and neurologic toxicity in melanoma patients during vemurafenib administration following anti-PD-1 therapy. Cancer Immunol Res 1(6): 373-377.

Kaufman H, Lutzky J, Clark J, Margolin KA, Lawson DH, Amin A, Collichio FA, Pecora A, Urba WJ, Bennett KL, McDermott DF (2013) Safety and efficacy of ipilimumab in melanoma patients who received prior immunotherapy on phase III study MDX010-020. J Clin Oncol. (suppl; abstr 9050).

Khoja L, Butler MO, Chappell MA, Hogg D, Joshua AM (2015) Increased treatment-related toxicity subsequent to an anti-PD-1 agent. Curr Oncol 22(4): 320-322.

Larkin J, Chiarion-Sileni V, Gonzalez R, Grob JJ, Cowey CL, Lao CD, Schadendorf D, Schadendorf, Dummer R, Smylie M, Rutkowski P, Ferrucci PF, Hill A, Wagstaff J, Carlino MS, Haanen JB, Maio M, Marquez-Rodas I, McArthur GA, Ascierto PA, Long GV, Callahan MK, Postow MA, Grossmann K, Sznol M, Dreno B, Bastholt L, Yang A, Rollin LM, Horak C, Hodi FS, Wolchok JD (2015) Combined nivolumab and ipilimumab or monotherapy in untreated melanoma. $N$ Engl J Med 373: 23-34

Merck (2014) KEYTRUDA® Prescribing Information http:// www.merck.com/product/usa/pi_circulars/k/keytruda/keytruda_pi.pdf.

Robert C, Long GV, Brady B, Dutriaux C, Maio M, Mortier L, Hassel JC, Rutkowski P, McNeil C, Kalinka-Warzocha E, Savage KJ, Hernberg MM, Lebbé C, Charles J, Mihalcioiu C, Chiarion-Sileni V, Mauch C, Cognetti F, Arance A, Schmidt H, Schadendorf D, Gogas H, Lundgren-Eriksson L, Horak C, Sharkey B, Waxman IM, Atkinson V, Ascierto PA (2015a) Nivolumab in previously untreated melanoma without BRAF mutation. N Engl J Med 372(4): 320-330.

Robert C, Schachter J, Long GV, Arance A, Grob JJ, Mortier L, Daud A, Carlino MS, McNeil C, Lotem M, Larkin J, Lorigan P, Neyns B, Blank CU, Hamid O, Mateus C, Shapira-Frommer R, Kosh M, Zhou H, Ibrahim N, Ebbinghaus S, Ribas A (2015b) Pembrolizumab versus ipilimumab in advanced melanoma. $N$ Engl J Med 372(26): 2521-2532.

Robert C, Thomas L, Bondarenko I, O’Day S, Weber J, Garbe C, Lebbe C, Baurain JF, Testori A, Grob JJ, Davidson N, Richards J, Maio M, Hauschild A, Miller WH, Gascon P, Lotem M, Harmankaya K, Ibrahim R, Francis S, Chen TT, Humphrey R, Hoos A, Wolchok JD (2011) Ipilimumab plus dacarbazine for previously untreated metastatic melanoma. N Engl J Med 364(26): 2517-2526.

Rotte A, Bhandaru M, Zhou Y, Mcelwee KJ (2015) Immunotherapy of melanoma: present options and future promises. Cancer Metastasis Rev 34(1): 115-128.

Schadendorf D, Hodi FS, Robert C, Weber JS, Margolin K, Hamid O, Patt D, Chen TT, Berman DM, Wolchok JD (2015) Pooled analysis of long-term survival data from phase II and phase III trials of ipilimumab in unresectable or metastatic melanoma. J Clin Oncol 33: 1889-1894.

Teng MWL, Ngiow SF, Ribas A, Smyth MJ (2015) Classifying cancers based on T-cell infiltration and PD-L1. Cancer Res 75: 2139-2145.

Topalian SL, Sznol M, McDermott DF, Kluger HM, Carvajal RD, Sharfman WH, Brahmer JR, Lawrence DP, Atkins MB, Powderly JD, Leming PD, Lipson EJ, Puzanov I, Smith DC, Taube JM, Wigginton JM, Kollia GD, Gupta A, Pardoll DM, Sosman JA, Hodi FS (2014) Survival, durable tumor remission, and long-term safety in patients with advanced melanoma receiving nivolumab. J Clin Oncol 32(10): 1020-1030.

Weber JS, Yang JC, Atkins MB, Disis ML (2015) Toxicities of immunotherapy for the practitioner. J Clin Oncol 33(18): 2092-2100.

Weber JS, Kähler KC, Hauschild A (2012) Management of immune-related adverse events and kinetics of response with ipilimumab. J Clin Oncol 30(21): 2691-2697.

Weber JS, Kudchadkar RR, Yu B, Gallenstein D, Horak CE, Inzunza HD, Zhao X, Martinez AJ, Wang W, Gibney G, Kroeger J, Eysmans C, Sarnaik AA, Chen YA (2013) Safety, efficacy, and biomarkers of nivolumab with vaccine in ipilimumab-refractory or -naive melanoma. J Clin Oncol 31(34): 4311-4318. 
Wolchok JD, Kluger H, Callahan MK, Postow MA, Rizvi NA, Lesokhin AM, Segal NH, Ariyan CE, Gordon RA, Reed K, Burke MM, Caldwell A,

Kronenberg SA, Agunwamba BU, Zhang X, Lowy I, Inzunza HD, Feely W, Horak CE, Hong Q, Korman AJ, Wigginton JM, Gupta A, Sznol M (2013) Nivolumab plus ipilimumab in advanced melanoma. N Engl J Med 369(2): 122-133.

Wolchok JD, Hoos A, O’Day S, Weber JS, Hamid O, Lebbé C, Maio M, Binder M, Bohnsack O, Nichol G, Humphrey R, Hodi FS (2009) Guidelines for the evaluation of immune therapy activity in solid tumors: immune-related response criteria. Clin Cancer Res 15(23): $7412-7420$

This work is published under the standard license to publish agreement. After 12 months the work will become freely available and the license terms will switch to a Creative Commons AttributionNonCommercial-Share Alike 4.0 Unported License. 